\title{
Echocardiographic Evaluation of Right Ventricular Function in ARDS Patients caused by COVID-19 pneumonia
}

\author{
Yunlong $\mathrm{Li}^{1}$, Junbo Zheng ${ }^{2}$, Yu Jin ${ }^{2}$, Rong Tang ${ }^{2}$, Ming $\mathrm{Li}^{2}$, Chunhong Xiu ${ }^{2}$, Qingqing \\ $\mathrm{Dai}^{2}$, Shu Zuo ${ }^{2}$, Huaiquan Wang ${ }^{2}$, Hongliang Wang ${ }^{2}$, Ming $\mathrm{Ye}^{2}$, Mingyan Zhao ${ }^{2}$, and \\ Kaijiang $\mathrm{Yu}^{2}$ \\ ${ }^{1}$ Second Affiliated Hospital of Harbin Medical University \\ ${ }^{2}$ Affiliation not available
}

July 16, 2020

\begin{abstract}
Backgound: To investigate the right heart function in COVID-19 (coronavirus disease 2019) patients with acute respiratory distress syndrome (ARDS). Methods: A retrospective analysis of 49 COVID-19 patients with ARDS was performed. Patients were divided into severe and critically severe group according to severity of illness. Age-matched healthy volunteers were recruited serving as a control group. The cardiac cavity diameters, tricuspid annular plane systolic excursion (TAPSE), tricuspid valve regurgitation pressure gradient biggest (TRPG), pulmonary arterial systolic pressure (PASP), maximum inferior vena cava diameter (IVCmax) and minimum diameter (IVCmin), and inferior vena cava collapse index (ICV-CI) were measured using echocardiography. Results: We found the TAPSE was significantly decreased in pneumonia patients compared to healthy subjects $(\mathrm{P}<0.0001)$, which was significantly lower in critically severe patients $(\mathrm{P}=0.0068)$. TAPSE was less than $17 \mathrm{~mm}$ in 3 $(8.6 \%)$ severe and $5(35.7 \%)$ critically severe patients. There were no significant differences in PASP and TRPG between severe and critically severe patients. The IVCmax and IVCmin were significantly increased in critically severe patients compared to healthy subjects and severe patients $(\mathrm{P}<0.01)$, whereas ICV-CI was significantly decreased $(\mathrm{P}<0.05)$. COVID-19 patients had significantly larger right atrium and ventricle than healthy controls $(\mathrm{P}<0.01)$. The left ventricular ejection fraction $(\mathrm{LVEF})$ in critically severe patients was significantly lower than that in severe patients and healthy controls $(\mathrm{P}<0.05)$. Conclusion: Right ventricular function is impaired in critically severe COVID-19 patients. The assessment and protection of the right heart function in COVID-19 patients should be strengthened.
\end{abstract}

\section{Background}

Coronavirus disease 2019 (COVID-19) pneumonia, caused by the SARS coronavirus 2 (SARS-CoV-2), has spread rapidly all over the world $[1,2]$. It can induce systemic multiple organ damage, especially the lungs [3]. It is reported that $26.1 \%$ of patients required admission to an intensive care unit (ICU) and $61.1 \%$ of them developed acute respiratory distress syndrome (ARDS) [4]. Moreover, the incidence of ARDS among the patients who died of COVID-19 pneumonia was $81 \%$, however, which was $45 \%$ in survivors [5].

We have already known that ARDS is often accompanied by right ventricular (RV) dysfunction and the incidence is about $20-30 \%$, and RV dysfunction is an important determinant of mortality in ARDS patients [6-8]. However, the incidence of RV dysfunction in patients with ARDS caused by COVID-19 pneumonia is unclear. At present, echocardiography is still the prefered choice for evaluating RV function of patients with ARDS, with the advantages of bedside, non-invasive and convenient. Tricuspid annular plane systolic excursion (TAPSE) is widely used as a quantitative index to evaluate RV function, which is highly correlated with the mortality of ARDS patients, thus providing a good parameter for us to interpret RV function in COVID-19 patients [9]. 
In this study, we sought to determine the incidence of RV dysfunction using echocardiography in a cohort of patients with ARDS caused by COVID-19 pneumonia.

\section{Methods}

\section{Study population}

We retrospectively analyzed 49 patients with ARDS caused by COVID-19 pneumonia (age [?]18 years) who were admitted to the ICU of the First Affiliated Hospital of Harbin Medical University from February to March 2020. The novel coronavirus nucleic acid test results were positive in all patients. The diagnosis of ARDS was based on the Berlin definition [10]. According to the Guidelines for diagnosis and treatment of novel coronavirus pneumonia (Trial Version 5) [11], all selected patients were divided into severe and critically severe group. Patients with polypnea (respiratory rate [?] 30 times/min), decreased oxygen saturation ([?] $93 \%$ at rest), $\mathrm{PaO}_{2} / \mathrm{FiO}_{2}$ [?] $300 \mathrm{mmHg}$ or exacerbation of lung imaging more than $50 \%$ within $24-48$ hours were classified as severe type, and those with respiratory failure requiring mechanical ventilation, shock or other organ failure were classified as critically severe type. Patients with no echocardiographic windows, or who had congenital heart disease, cardiac valvular disease or acute myocardial infarction were excluded. All 49 patients were enrolled for final analysis, including 35 severe and 14 critically severe cases. Age, gender, underlying disease, APACHE II score within 24 hours of hospitalization, heart rate (HR) and mean arterial pressure (MAP) during echocardiographic examination were recorded. Age-matched healthy volunteers (n $=25)$ were recruited serving as a control group.

\section{Echocardiography}

Echocardiography assessment was performed in all patients within $48 \mathrm{~h}$ of ICU admission by the same echocardiographer using a commercially available system (Vivid E9, GE, Inc.) equipped with a 2.5-4MHz transducer (M5S probe). All patients were examined in a calm or sedated state. All echocardiographic parameters were evaluated according to American Society of Echocardiography (ASE) guidelines [12].

\section{RV function assessment}

TAPSE was defined as the peak excursion of tricuspid annulus from end-diastole to end-systole, and was measured from the apical four-chamber view by placing the M-mode cursor through the lateral tricuspid annulus. TAPSE less than $17 \mathrm{~mm}$ indicated RV dysfunction [12].

The diameter changes of inferior vena cava (IVC) during the respiratory cycle were measured from the IVC long axis view by M-mode. The minimum diameter of the inferior vena cava (IVCmin) was measured in the inspiratory phase and the maximum diameter of the inferior vena cava (IVCmax) was measured in the expiratory phase when breathing spontaneously. The inferior vena cava collapse index (ICV-CI) was calculated by the formula of (IVCmax-IVCmin) / IVCmax $\times 100 \%$.

The diameter of pulmonary artery (PAD) was measured from parasternal short-axis view by two-dimensional echocardiography. Tricuspid valve regurgitation pressure gradient biggest (TRPG) was measured and pulmonary arterial systolic pressure (PASP) was estimated indirectly by tricuspid regurgitation velocity with the addition of right atrial pressure, assuming no significant right ventricular outflow tract (RVOT) obstruction.

\section{Cardiac diameters and left ventricular function}

By two-dimensional echocardiography, left ventricular end-diastolic dimension (LVEDD) was measured from the parasternal long-axis view, while the right atrial diameter (RAD) and right ventricular diameter (RVD) were measured from the apical four-chamber view. The sampling volume was placed above the aortic valve orifice to obtain the aortic valve blood flow spectrum and measure the aortic valve velocity (AV) from the apical five-chamber view. A modified Simpson's method was used to measure left ventricular end-diastolic volume (EDV) and end-systolic volume (ESV). Left ventricular ejection fraction(LVEF) was calculated as followed: $\mathrm{LVEF}=(\mathrm{EDV}-\mathrm{ESV}) / \mathrm{EDV}$.

\section{Statistical analysis}


The statistical analysis was performed using GraphPad Prism 6. Categorical variables are reported as frequencies and percentages. Continuous variables are reported as mean \pm standard deviation (SD). Categorical variables were compared using Chi square test. For comparison between severe and critically severe patients, the Mann-Whitney U test was used. One-way ANOVA followed by Tukey's test was used for statistical comparisons between healthy subjects, severe and critically severe group.

\section{Results}

\section{Patient characteristics}

Among all patients enrolled, $35(71.4 \%)$ were severe type and $14(28.6 \%)$ were critically severe type. There were $22(62.9 \%)$ patients with mild and $13(37.1 \%)$ with moderate ARDS in the severe patients, $4(28.6 \%)$ with moderate and $10(71.4 \%)$ with severe ARDS in the critically severe patients. The clinical characteristics of patients are shown in Table 1.

There was no significant difference in age between COVID-19 pneumonia patients and healthy donors $(\mathrm{P}>$ 0.05). Critically severe patients had significantly higher APACHE II score $(\mathrm{P}=0.0001)$ and lower $\mathrm{PaO}_{2} / \mathrm{FiO}_{2}$ compared to severe patients $(\mathrm{P}<0.0001)$. The HR in critically severe patients was significantly increased compared to the healthy group $(\mathrm{P}=0.0056)$, and the MAP was significantly decreased than that in the healthy group and critically severe group $(\mathrm{P}<0.01)$. In critically severe patients, $11(78.6 \%)$ cases received invasive mechanical ventilation and $8(57.1 \%)$ were treated with vasoactive drugs. There was no statistically significant difference in comorbidity between severe and critically severe patients $(\mathrm{P}>0.05)$.

\section{RV function}

As shown in Figure 1, a normal TAPSE was recorded in all healthy subjects, but the TAPSE was significantly decreased in patients with COVID-19 pneumonia. Further, the TAPSE was significantly lower in critically severe patients than in severe patients $(\mathrm{P}=0.0128) .3(8.6 \%)$ severe and $5(35.7 \%)$ critically severe patients had a reduced TAPSE $(<17 \mathrm{~mm})$.

The echocardiographic parameters of RV function in patients with COVID-19 pneumonia are shown in Table 2. Tricuspid regurgitation was present in $21(60.0 \%)$ severe and $13(92.9 \%)$ critically severe patients, and there were no statistically significant differences in PASP and TRPG between the two groups $(\mathrm{P}>0.05)$. Patients with COVID-19 pneumonia had a significantly higher PAD than healthy subjects $(\mathrm{P}<0.0001)$. The IVCmax and IVCmin were significantly increased in critically severe patients than in healthy subjects and severe patients $(\mathrm{P}<0.01)$, whereas ICV-CI was significantly decreased $(\mathrm{P}<0.05)$.

\section{The diameters of cardiac cavity and LVEF}

The diameters of cardiac cavity and other echocardiographic parameters are shown in Table 3. Patients with COVID-19 pneumonia had significantly larger right atrium and right ventricle than healthy subjects $(\mathrm{P}<$ 0.01 ), and there were no statistically significant difference in RAD and RVD between severe and critically severe patients $(\mathrm{P}>0.05)$. Critically severe patients had a significantly lower LVEF than healthy subjects and severe patients $(\mathrm{P}<0.05)$.

\section{Discussion}

Our results demonstrated that RV dysfunction is an important clinical feature in COVID-19 pneumonia patients with ARDS, which is related to the severity of COVID-19 pneumonia and ARDS. Bedside echocardiography allows an early integrated assessment of the RV function through the evaluation of TAPSE. The echocardiographic parameters of RV function and cardiac cavity in COVID-19 pneumonia patients show abnormity to different extents compared to healthy subjects.

Viral pneumonia is the one of the most common causes for ARDS and is associated with high morbidity and mortality worldwide, such as SARS, H1N1 flu and so on [13-15]. RV dysfunction is frequently presented in these patients, and leads to left-right asymmetry of the heart, which can worsen hemodynamic stability and is associated with a poorer prognosis $[6,16,17]$. In our study, a higher incidence of RV dysfunction was 
found in critically severe group who had more severe ARDS patients, which is similar to that in the severe ARDS population caused by SARS and H1N1 viruses [15, 18, 19]. Thus, RV dysfunction is common in viral pneumonia patients, especially in patients with severe ARDS.

TAPSE, as the most commonly used index for evaluating RV function, has been widely used in patients with ARDS [20]. A TAPSE less than $17 \mathrm{~mm}$ was recommended as a major parameter of RV dysfunction by ASE guidelines. In present study, we found the TAPSE in patients with COVID-19 pneumonia was significantly decreased compared to healthy people, and the proportion of patients with TAPSE $<17 \mathrm{~mm}$ was significantly higher in critically severe patients $(35.7 \%)$ than that in severe patients $(8.6 \%)$, indicating that there was a correlation between the occurrence of RV dysfunction and the severity of COVID-19 pneumonia. Patients in critically severe group appeared to be more severely ill in terms of APACHE II, decreased MAP and increased HR. Moreover, invasive ventilation and vasopressors are frequently required in these patients.

The function of the RV is to keep proper pulmonary perfusion pressure and systemic venous pressure to maintain normal blood flow [21]. The main factors affecting the RV function in patients with ARDS are impaired RV contraction and increased pulmonary vascular resistance. It is known that the increase of pulmonary vascular resistance may be induced by vasoconstrictor, hypoxemia, hypercapnia, acidosis and so on, which cause the deterioration of right heart structure and function [22-24]. Recent studies have shown that the clinical characteristics of severe and critically severe COVID-19 pneumonia patients are severe hypoxemia, hypercapnia and microthrombosis, which may lead to the increase of pulmonary vascular resistance [3-5].

In our study, the values of TRPG and PASP were higher than normal ranges and increased in COVID19 pneumonia patients. Although the majority of patients in critically severe group received mechanical ventilation, there were no significant differences in TRPG and PASP between the severe and critically severe patients. This may be because pulmonary vascular resistance is reduced by positive pressure mechanical ventilation and is not seriously deteriorated in the early stages of the disease [25-27]. The right heart had a poor ability to regulate pressure load [16]. In this study, the right heart cavity and pulmonary artery were enlarged soon after the increase of pulmonary vascular resistance in patients with COVID-19 pneumonia.

Patients with critically severe COVID-19 pneumonia are often accompanied by organ dysfunction and shock. The results of the present study have shown that both RV and left ventricular systolic function in critically severe patients with COVID-19 pneumonia are impaired. The hemodynamic instability of decreased LVEF and MAP is associated with more severe RV dysfunction, which present significantly decreased TAPSE, enhanced diameters of IVC and reduced ICV-CI. Therefore, the monitoring and protection of right ventricular function need to be strengthened in the management of patients with COVID-19 pneumonia. There are limitations in the present study. This is a retrospective study with limited sample size. The results need to be further confirmed by prospective clinical studies. In addition, although the assessments of echocardiography were performed by ultrasonologist, it became more challenged and difficult than usual to obtain parameters since three-level isolation and protection measures must be taken when contacting patients with COVID-19 pneumonia. However, in this case, echocardiography is the most important bedside examination method for evaluation of patients with COVID-19 pneumonia.

\section{Conclusions}

RV dysfunction is relatively common in patients with severe and critically severe COVID-19 pneumonia, which is related to the severity of illness. Early monitoring of RV function is very important for these patients. It is suggested that bedside echocardiography might be used as an objective examination in the evaluation of RV function in the COVID-19 pneumonia patients with ARDS. TAPSE may serve as a quantitative indicator of early detection of RV dysfunction. These findings are helpful to further improve the clinical diagnosis and treatment strategies for patients with COVID-19 pneumonia.

\section{Abbreviations}

ARDS: Acute respiratory distress syndrome; ASE: American society of echocardiography; AV: Aortic valve 
velocity; COVID-19: Coronavirus disease 2019; EDV: End-diastolic volume; ESV: End-systolic volume; HR: Heart rate; ICU: Intensive care unit; ICV-CI: Inferior vena cava collapse index; IVC: Inferior vena cava;IVCmax: Maximum diameter of the inferior vena cava; IVCmin: Minimum diameter of the inferior vena cava; LVEDD: Left ventricular end-diastolic dimension; LVEF: Left ventricular ejection fraction; MAP: Mean arterial pressure; PAD: Diameter of pulmonary artery; PASP: Pulmonary arterial systolic pressure; RAD: Right atrial diameter; RV: Right ventricular; RVD: Right ventricular diameter; RVOT: Right ventricular outflow tract; SARS-CoV-2: SARS coronavirus 2; SD: Standard deviation; TAPSE: Tricuspid annular plane systolic excursion; TRPG: Tricuspid valve regurgitation pressure gradient biggest.

\section{Declarations}

Ethics approval and consent to participate: The study was approved by the Second Affiliated Hospital of Harbin Medical University (KY2020-015).

Consent for publication: Written informed consent for publication was obtained from all participants.

Availability of data and materials: All data generated or analysed during this study are included in this published article.

Competing interests: The authors declare that they have no competing interests.

Funding: None to disclose

Authors' contributions: Yunlong Li, Ming Li, Rong Tang collected the epidemiological and clinical data. Huaiquan Wang, Ming Ye, Hongliang Wang guided the study design. Yunlong Li, Junbo Zheng, Yu Jin, Qingqing Dai, Shu Zuo, and Chunhong Xiu summarized all data. Yunlong Li, Junbo Zheng and Chunhong Xiu drafted the manuscript. Kaijing Yu, Ming Ye and Mingyan Zhao revised the final manuscript. All authors read and approved the final manuscript.

Acknowledgements: We thank all patients and healthy volunteers involved in the study.

\section{References}

1. Li Q, Guan X, Wu P, Wang X, Zhou L, Tong Y, et al. Early Transmission Dynamics in Wuhan, China, of Novel Coronavirus-Infected Pneumonia. N Engl J Med. 2020;382:1199-207.

2. World Health Organization. Coronavirus Disease (COVID-2019) Situation Reports. Update on 24:00 of Apr 2. Available online: https://http://www.who.int/emergencies/diseases/novel-coronavirus2019/situation-reports/.

3. Wu Z, McGoogan JM. Characteristics of and Important Lessons From the Coronavirus Disease 2019 (COVID-19) Outbreak in China: Summary of a Report of 72314 Cases From the Chinese Center for Disease Control and Prevention. JAMA. 2020.

4. Yang X, Yu Y, Xu J, Shu H, Xia J, Liu H, et al. Clinical course and outcomes of critically ill patients with SARS-CoV-2 pneumonia in Wuhan, China: a single-centered, retrospective, observational study. Lancet Respir Med. 2020.

5. Wang D, Hu B, Hu C, Zhu F, Liu X, Zhang J, et al. Clinical Characteristics of 138 Hospitalized Patients With 2019 Novel Coronavirus-Infected Pneumonia in Wuhan, China. JAMA. 2020.

6. Repesse X, Charron C, Vieillard-Baron A. Acute respiratory distress syndrome: the heart side of the moon. Curr Opin Crit Care. 2016;22:38-44.

7. Mekontso Dessap A, Boissier F, Charron C, Begot E, Repesse X, Legras A, et al. Acute cor pulmonale during protective ventilation for acute respiratory distress syndrome: prevalence, predictors, and clinical impact. Intensive Care Med. 2016;42:862-70. 
8. Boissier F, Katsahian S, Razazi K, Thille AW, Roche-Campo F, Leon R, et al. Prevalence and prognosis of cor pulmonale during protective ventilation for acute respiratory distress syndrome. Intensive Care Med. 2013;39:1725-33.

9. Wadia SK, Shah TG, Hedstrom G, Kovach JA, Tandon R. Early detection of right ventricular dysfunction using transthoracic echocardiography in ARDS: a more objective approach. Echocardiography. 2016;33:18749 .

10. Force ADT, Ranieri VM, Rubenfeld GD, Thompson BT, Ferguson ND, Caldwell E, et al. Acute respiratory distress syndrome: the Berlin Definition. JAMA. 2012;307:2526-33.

11. National Health Commission of the People's Republic of China. Guidelines for diagnosis and treatment of novel coronavirus pneumonia (Trial Version 5) 2020. http://www.nhc.gov.cn/yzygj/ .

12. Lang RM, Badano LP, Mor-Avi V, Afilalo J, Armstrong A, Ernande L, et al. Recommendations for cardiac chamber quantification by echocardiography in adults: an update from the American Society of Echocardiography and the European Association of Cardiovascular Imaging. Eur Heart J Cardiovasc Imaging. 2015;16:233-70.

13. Li SH, Hsieh MJ, Lin SW, Chuang LP, Lee CS, Chiu LC, et al. Outcomes of severe H1N1 pneumoniae: A retrospective study at intensive care units. J Formos Med Assoc. 2020;119:26-33.

14. Brown SM, Pittman J, Miller Iii RR, Horton KD, Markewitz B, Hirshberg E, et al. Right and left heart failure in severe H1N1 influenza A infection. Eur Respir J. 2011;37:112-8.

15. Viasus D, Pano-Pardo JR, Pachon J, Campins A, Lopez-Medrano F, Villoslada A, et al. Factors associated with severe disease in hospitalized adults with pandemic (H1N1) 2009 in Spain. Clin Microbiol Infect. 2011;17:738-46.

16. Arrigo M, Huber LC, Winnik S, Mikulicic F, Guidetti F, Frank M, et al. Right Ventricular Failure: Pathophysiology, Diagnosis and Treatment. Card Fail Rev. 2019;5:140-6.

17. Biswas A. Right heart failure in acute respiratory distress syndrome: An unappreciated albeit a potential target for intervention in the management of the disease. Indian J Crit Care Med. 2015;19:606-9.

18. Shorr AF, Zilberberg MD, Micek ST, Kollef MH. Viruses are prevalent in non-ventilated hospital-acquired pneumonia. Respir Med. 2017;122:76-80.

19. Yin Y, Wunderink RG. MERS, SARS and other coronaviruses as causes of pneumonia. Respirology. 2018;23:130-7.

20. Huang SJ, Nalos M, Smith L, Rajamani A, McLean AS. The use of echocardiographic indices in defining and assessing right ventricular systolic function in critical care research. Intensive Care Med. 2018;44:868-83.

Table 1. Clinical characteristics of healthy subjects and patients with COVID-19 pneumonia

\begin{tabular}{llll}
\hline & Healthy $(\mathrm{n}=25)$ & Severe patients $(\mathrm{n}=35)$ & Critically Severe $(\mathrm{n}=14)$ \\
Male & $12(48.0 \%)$ & $19(54.3 \%)$ & $6(42.9 \%)$ \\
$\mathrm{Age}$ & $62.2 \pm 8.2$ & $64.3 \pm 13.0$ & $65.6 \pm 14.6$ \\
$\mathrm{HR}$ & $72.5 \pm 8.9$ & $77.4 \pm 13.6$ & $88.0 \pm 22.6^{*}$ \\
$\mathrm{MAP}$ & $76.5 \pm 6.1$ & $90.7 \pm 12.1^{*}$ & $78.1 \pm 18.5^{\#}$ \\
$\mathrm{APACHE} \mathrm{II}$ & - & $6.2 \pm 3.7$ & $15.1 \pm 9.5{ }^{\#}$ \\
Mechanical ventilation & - & - & $11(78.6 \%)$ \\
$\mathrm{Vasoactive} \mathrm{agent}_{\mathrm{PaO}_{2}: \mathrm{FiO}_{2} \text { ratio }}$ & - & - & $8(57.1 \%)$ \\
$\mathrm{ARDS}$ & - & $259.1 \pm 136.8$ & $92.3 \pm 34.2$ \\
Mild & - & & \\
Moderate & - & $22(62.9 \%)$ & - \\
& & $13(37.1 \%)$ & $4(28.6 \%)$
\end{tabular}


Severe

Co-morbidities

Hypertension

Diabetes

Cardiovascular disease

-
-
-

$10(71.4 \%)$

$12(34.3 \%)$

$8(22.9 \%)$

$6(17.1 \%)$
$5(35.7 \%)$

$5(35.7 \%)$

$2(14.3 \%)$

Note: HR: heart rate; MAP: mean arterial pressure; APACHE II: Acute Physiology and Chronic Health Evaluation II; ARDS: acute respiratory distress syndrome. ${ }^{*} \mathrm{p}<0.05$ compared with healthy group; ${ }^{\#} \mathrm{p}<$ 0.05 compared with severe patients group.

Table 2. Echocardiographic parameters of right ventricular function

\begin{tabular}{lllll}
\hline & Healthy $(\mathrm{n}=25)$ & Severe patients $(\mathrm{n}=35)$ & Critically Severe $(\mathrm{n}=14)$ & Critically Severe $(\mathrm{n}=14)$ \\
PAD $(\mathrm{mm})$ & $20.2 \pm 2.9$ & $23.7 \pm 2.0^{*}$ & $25.3 \pm 4.6^{*}$ & \\
TRPG $(\mathrm{mmHg})$ & - & $25.3 \pm 5.2$ & $28.8 \pm 11.0$ & \\
PASP $(\mathrm{mmHg})$ & - & $29.8 \pm 4.8$ & $35.9 \pm 13.5$ \\
TAPSE $(\mathrm{mm})$ & $23.8 \pm 3.4$ & $20.4 \pm 2.4^{*}$ & $17.6 \pm 3.4^{*} \#$ \\
IVCmax $(\mathrm{mm})$ & $14.4 \pm 3.5$ & $14.0 \pm 2.0$ & $17.6 \pm 3.8^{*} \#$ \\
IVCmin $(\mathrm{mm})$ & $7.7 \pm 4.8$ & $6.2 \pm 2.1$ & $12.1 \pm 5.7^{*} \#$ \\
ICV-CI $(\%)$ & $50.4 \pm 22.3$ & $55.9 \pm 12.6$ & $34.1 \pm 24.8^{*} \#$ \\
\hline
\end{tabular}

Note: PAD: pulmonary artery diameter; TRPG: tricuspid valve regurgitation pressure gradientbiggest; PASP: pulmonary arterial systolic pressure; TAPSE: tricuspid annular plane systolic excursion; IVCmax: maximum diameter of the inferior vena cava; IVCmin: minimum diameter of the inferior vena cava; ICV-CI: inferior vena cava collapse index. ${ }^{*} \mathrm{p}<0.05$ compared with healthy group; ${ }^{\#} \mathrm{p}<0.05$ compared with severe patients group

Table 3. Cardiac diameters and left ventricular function

\begin{tabular}{llll}
\hline & Healthy $(\mathrm{n}=25)$ & Severe patients $(\mathrm{n}=35)$ & Critically Severe $(\mathrm{n}=14)$ \\
LVEDD $(\mathrm{mm})$ & $43.6 \pm 3.5$ & $45.6 \pm 2.9$ & $44.3 \pm 3.0$ \\
RVD $(\mathrm{mm})$ & $17.6 \pm 3.1$ & $34.7 \pm 3.3^{*}$ & $34.6 \pm 3.3^{*}$ \\
RAD $(\mathrm{mm})$ & $30.4 \pm 3.4$ & $32.7 \pm 2.3^{*}$ & $34.1 \pm 3.6^{*}$ \\
LVEF $(\%)$ & $64.8 \pm 4.0$ & $63.9 \pm 5.0$ & $59.4 \pm 8.4 * \#$ \\
AV $(\mathrm{cm} / \mathrm{s})$ & $117.4 \pm 9.3$ & $112.7 \pm 16.1$ & $112.1 \pm 16.3$ \\
\hline
\end{tabular}

Note: LVEDD: left ventricular end-diastolic dimension; RVD: right ventricular diameter; RAD: right atrial diameter; LVEF: left ventricular ejection fraction; AV: aortic valve velocity. ${ }^{*} \mathrm{p}<0.05$ compared with healthy group; \# p $<0.05$ compared with severe patients group.

\section{FIGURE LEGENDS}

Fig. 1 Values of tricuspid annular plane systolic excursion (TAPSE) for individual COVID-19 pneumonia patients and healthy subjects. 


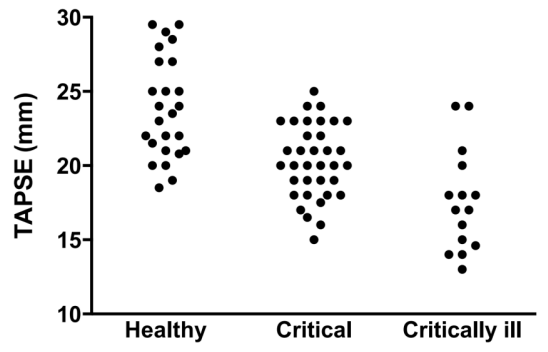

Fig. 1 